\title{
Effector $\mathrm{V} \gamma 9 \mathrm{~V} \delta 2 \mathrm{~T}$ cell response to congenital Toxoplasma gondii infection
}

\author{
Ling Ma, ${ }^{1,2,3}$ Maria Papadopoulou, ${ }^{1,2,3}$ Martin Taton, ${ }^{2,3}$ Francesca Genco, ${ }^{4}$ Arnaud Marchant, ${ }^{2,3}$ \\ Valeria Meroni, ${ }^{4,5}$ and David Vermijlen ${ }^{1,2,3}$ \\ 'Department of Pharmacotherapy and Pharmaceutics, ${ }^{2}$ Institute for Medical Immunology, and ${ }^{3}$ ULB Center for Research in \\ Immunology, Université Libre de Bruxelles (ULB), Brussels, Belgium. ${ }^{4}$ IRCCS San Matteo Polyclinic, Pavia, Italy. ${ }^{5}$ Molecular \\ Medicine Department, University of Pavia, Italy.
}

\begin{abstract}
A major $\gamma \delta$ T cell population in human adult blood are the $\mathrm{V} \gamma 9 \mathrm{~V} \delta 2 \mathrm{~T}$ cells that are activated and expanded in a TCR-dependent manner by microbe-derived and endogenously derived phosphorylated prenyl metabolites (phosphoantigens). $\mathrm{V} \gamma \mathbf{9 V} \delta 2 \mathrm{~T}$ cells are also abundant in human fetal peripheral blood, but compared with their adult counterparts they have a distinct developmental origin, are hyporesponsive toward in vitro phosphoantigen exposure, and do not possess a cytotoxic effector phenotype. In order to obtain insight into the role of $\mathrm{V} \gamma 9 \mathrm{~V} \delta 2 \mathrm{~T}$ cells in the human fetus, we investigated their response to in utero infection with the phosphoantigenproducing parasite Toxoplasma gondii ( $T$. gondii). $\mathrm{V} \gamma \mathrm{gV} \delta 2 \mathrm{~T}$ cells expanded strongly when faced with congenital $T$. gondii infection, which was associated with differentiation toward potent cytotoxic effector cells. The $\mathrm{V} \gamma \mathrm{gV} \delta 2 \mathrm{~T}$ cell expansion in utero resulted in a fetal footprint with public germline-encoded clonotypes in the $\mathrm{V} \gamma \boldsymbol{g} \mathbf{V} \delta 2 \mathrm{TCR}$ repertoire 2 months after birth. Overall, our data indicate that the human fetus, from early gestation onward, possesses public $\mathrm{V} \gamma 9 \mathrm{~V} \delta 2 \mathrm{~T}$ cells that acquire effector functions following parasite infections.
\end{abstract}

Conflict of interest: The authors have declared that no conflict of interest exists.

Copyright: ( ${ }^{2} 2021$, Ma et al. This is an open access article published under the terms of the Creative Commons Attribution 4.0 International License.

Submitted: March 11, 2020

Accepted: July 7, 2021

Published: August 23, 2021

Reference information: /CI Insight. 2021;6(16):e138066.

https://doi.org/10.1172/jci.

insight.138066.

\section{Introduction}

$\gamma \delta$ T cells are T lymphocytes that express a TCR containing $\gamma$ and $\delta$ chains, instead of $\alpha$ and $\beta$ chains as in the conventional $\mathrm{CD}^{+}$and $\mathrm{CD}^{+} \alpha \beta$ T cells. As $\alpha \beta$ T cells, $\gamma \delta \mathrm{T}$ cells have been conserved for more than 450 million years of evolution and play an important role against infection and cancer (1-3). A major $\gamma \delta \mathrm{T}$ cell population in human adult blood are the $\mathrm{V} \gamma 9 \mathrm{~V} \delta 2 \mathrm{~T}$ cells that are defined by the expression of a TCR containing the $\gamma$ chain variable region $9(\mathrm{~V} \gamma 9$, TRGV9) and the $\delta$ chain $\mathrm{V}$ region 2 (V82, TRDV2). They express a potent cytotoxic effector phenotype and are activated and expanded in a TCR-dependent manner by microbe- and host-derived phosphorylated prenyl metabolites (phosphorylated Ags, or phosphoantigens), derived from the isoprenoid metabolic pathway (4-6). The prototypical example of a microbial phosphoantigen is (E)-4-hydroxy-3-methyl-but-2-enyl pyrophosphate (HMBPP) produced by the 2-Cmethyl-D-erythritol 4-phosphate (MEP) pathway of isoprenoid synthesis (also known as the nonmevalonate pathway) that is present in bacteria and in protozoan parasites of the phylum Apicomplexa (4). The recognition of phosphoantigens allows adult $\mathrm{V} \gamma 9 \mathrm{~V} \delta 2 \mathrm{~T}$ cells to develop potent antimicrobial and anticancer immune responses (2, 4, 7-9). While $\mathrm{V} \gamma 9 \mathrm{~V} \delta 2 \mathrm{~T}$ cells are also abundant in fetal peripheral blood, they are hyporesponsive toward phosphoantigen stimulation in vitro, they are highly regulated by programmed cell death protein 1 , and they do not show a cytotoxic effector phenotype (10-15). These features are likely related to (tolerance) requirements of the fetal immune system, which involves a distinct thymic development (16-18).

During development of T cells in the thymus, TCR gene rearrangements take place where single V (variable), D (diversity; only for TRD), and J (joining) gene segments join to form a final chain ( $\alpha, \beta, \gamma$, or $\delta$ ). The variability created during the $\mathrm{V}(\mathrm{D}) \mathrm{J}$ recombination is significantly enhanced by the junctional diversity, which comprises (a) incorporation of palindromic sequences ("P nucleotides"), (b) the introduction of additional random nucleotides ("N additions") in the junction by the terminal deoxynucleotidyl transferase (TdT enzyme), and (c) deletion of nucleotides (by exonuclease) (19). The pairing of a single $\gamma(\mathrm{TRG})$ with a $\delta$ (TRD) chain will give rise to the final TCR expressed on the surface of the $\gamma \delta$ T cell. The most variable domain, usually responsible for antigen recognition, is found in the complementarity determining region 3 (CDR3) and is the region most often analyzed. In human, $\gamma \delta \mathrm{T}$ cells are the most 
abundant lymphoid population in the embryonic thymus in early gestation, with a shift around gestation week 11, when they decrease significantly and the $\alpha \beta$ T cells take the lead (20). The very first $\gamma \delta$ T cell population to arise in humans is the $\mathrm{V} \gamma 9 \mathrm{~V} \delta 2$ subset, detected in the embryonic (prethymic) liver from as early as 5 to 6 weeks of gestation (21) and in fetal thymus after 8 weeks of gestation $(17,22)$, which is then likely to exit the thymus toward the fetal blood $(10,19)$. We have recently shown that fetal and adult $\mathrm{V} \gamma 9 \mathrm{~V} \delta 2$ $\mathrm{T}$ cells are generated by the thymus at different time points in life, and we have identified key differences between the TCR repertoire of fetal and adult blood $\mathrm{V} \gamma 9 \mathrm{~V} \delta 2 \mathrm{~T}$ cells, including the very low number of $\mathrm{N}$ additions in the fetal V $\gamma 9 \mathrm{~V} \delta 2$ TCR repertoire (17).

Toxoplasma gondii ( $T$. gondii) is an obligate intracellular protozoan that belongs to the phylum Apicomplexa and, thus, produces the potent phosphoantigen HMBPP. It infects up to one-third of the world's population, and infection acquired during pregnancy (congenital infection) may cause severe damage to the fetus $(23,24)$. Previous studies indicate that $\gamma \delta \mathrm{T}$ cells play a role in the immune response against $T$. gondii. Indeed, mice depleted of $\gamma \delta \mathrm{T}$ cells are more susceptible to $T$. gondii infection (25), human adult V $\gamma 9 \mathrm{~V} \delta 2 \mathrm{~T}$ cells are expanded upon acute toxoplasmosis, and an in vitro study showed that $\mathrm{V} \gamma 9 \mathrm{~V} \delta 2 \mathrm{~T}$ cells are activated by incubation with $T$. gondii-infected cells, resulting in the killing of the infected cells (26). While some changes have been observed in cord blood V $\gamma 9 \mathrm{~V} \delta 2 \mathrm{~T}$ cells in placental malaria (27), it remains unclear whether fetal $\mathrm{V} \gamma 9 \mathrm{~V} \delta 2 \mathrm{~T}$ cells can develop immune responses against pathogens that cross through the placenta, such as in congenital toxoplasmosis ( 13 , $15,28,29)$. We found that fetal $\mathrm{V} \gamma 9 \mathrm{~V} \delta 2 \mathrm{~T}$ cells expanded strongly and differentiated toward potent killer effector cells in infants with congenital $T$. gondii infection.

\section{Results}

Study population. Pregnant mothers with primary T. gondii infection were enrolled in this study. In order to address the fetal/newborn immune response toward T. gondii infection, blood samples from their infants were collected. Fifteen out of 74 infants $(20 \%)$ were diagnosed with congenital toxoplasmosis $\left(\mathrm{Toxo}^{+}\right)$. The $\mathrm{Toxo}^{+}$infants were age matched with their noninfected (Toxo-) counterparts (Figure 1A; age of Toxo ${ }^{-}$versus Toxo ${ }^{+}$subjects: $P=0.0688$ ). For most infants ( 66 out of 74$), 1$ blood sample was collected, for 7 infants 2 blood samples, and for 1 infant 3 blood samples were collected between birth and 2 years of age (Figure 1A). Clinical characteristics, such as age at the moment of diagnosis and treatment schedules of the Toxo infants, can be found in Table 1. All deliveries (of $\mathrm{Toxo}^{+}$and $\mathrm{Toxo}^{-}$infants) were term deliveries ( $>37$ weeks gestation). No clinical problems were observed in the Toxo- infants.

$V \gamma 9 V \delta 2$ T cells expand strongly upon congenital T. gondii infection. First, we determined the percentage of $\gamma \delta$ $\mathrm{T}$ cells (out of total $\mathrm{T}$ cells) and found that they were significantly increased in Toxo ${ }^{+}$infants (Figure 1A). As can be seen from the plotting of the percentage of $\gamma \delta \mathrm{T}$ cells versus the age of the infants, this higher percentage of $\gamma \delta \mathrm{T}$ cells was especially evident early after birth (0- to 2-month-old newborns, Figure 1A). At older ages ( $>2$ months), the difference between $\mathrm{Toxo}^{+}$and $\mathrm{ToxO}^{-}$infants diminished, mainly because the percentage of $\gamma \delta \mathrm{T}$ cells started to increase in the Toxo- group (Figure 1A). The increase in $\gamma \delta \mathrm{T}$ cells' percentages early after birth was not associated with an increased expression of the proliferation marker Ki-67 (Figure 1B). To ascertain that this high percentage of $\gamma \delta \mathrm{T}$ cells was not due to changes in $\alpha \beta \mathrm{T}$ cells, we quantified absolute numbers and confirmed that the $\gamma \delta$ T cells were increased, while no change could be detected in the $\alpha \beta$ T cell compartment (Supplemental Figure 1, A and B; supplemental material available online with this article; https://doi.org/10.1172/jci.insight.138066DS1).

Next, we investigated the $\mathrm{V} \gamma 9 \mathrm{~V} \delta 2$ subset more specifically and compared it with other $\gamma \delta$ subsets. Using antibodies specifically against the $\mathrm{V} \gamma 9$ and $\mathrm{V} \delta 2$ chain (combined with CD3 and pan- $\gamma \delta$ antibodies), we could delineate 4 different populations: $\mathrm{V} \gamma 9^{+} \mathrm{V} \delta 2^{+}, \mathrm{V} \gamma 9^{+} \mathrm{V} \delta 2^{-}, \mathrm{V} \gamma 9^{-} \mathrm{V} \delta 2^{+}$, and $\mathrm{V} \gamma 9^{-} \mathrm{V} \delta 2^{-} \gamma \delta$ T cells (Figure 2, Supplemental Figure 1C, and Supplemental Figure 2). The increase in newborn $\gamma \delta \mathrm{T}$ cells upon congenital T. gondii infection was highly restricted to the $\mathrm{V} \gamma 9^{+} \mathrm{V} \delta 2^{+}$subset (Figure 2, Supplemental Figure 1C, and Supplemental Figure 2); the other subsets did not show different percentages or numbers compared to Toxo ${ }^{-}$newborns, including the more abundant $\mathrm{V} \gamma 9^{-} \mathrm{V} \delta 2^{-} \gamma \delta \mathrm{T}$ cell subset (Figure $2 \mathrm{~B}$, Supplemental Figure $1 C$, and Supplemental Figure 2). Gating on newborn V $\gamma 9 \mathrm{~V} \delta 2 \mathrm{~T}$ cells, again no increase in the Ki-67 proliferation marker could be observed between $\mathrm{Toxo}^{+}$and $\mathrm{Toxo}^{-}$subjects (Supplemental Figure 3), indicating that the proliferation of $\mathrm{V} \gamma 9 \mathrm{~V} \delta 2 \mathrm{~T}$ cells had already occurred in utero, accounting for the higher percentage and number of newborn $\mathrm{V} \gamma 9 \mathrm{~V} \delta 2 \mathrm{~T}$ cells in the $\mathrm{Toxo}^{+}$compared with the Toxo ${ }^{-}$group (Figure 2, Supplemental Figure 1C, and Supplemental Figure 2). 
A

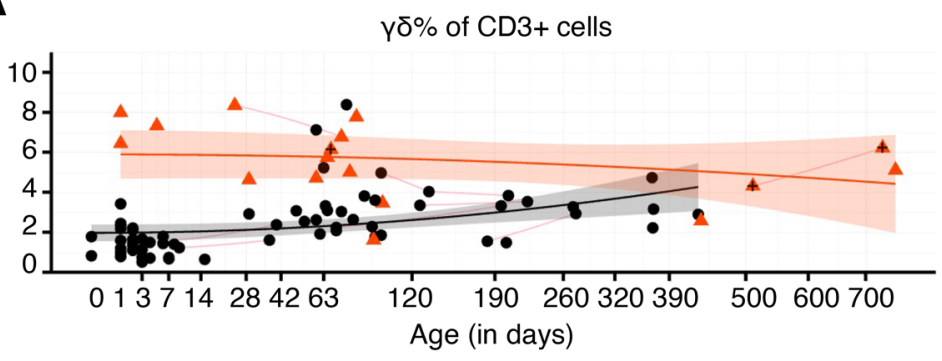

all: $P=2.7 \mathrm{e}-06$

wo retinitis: $P=1.4 \mathrm{e}-05$

B

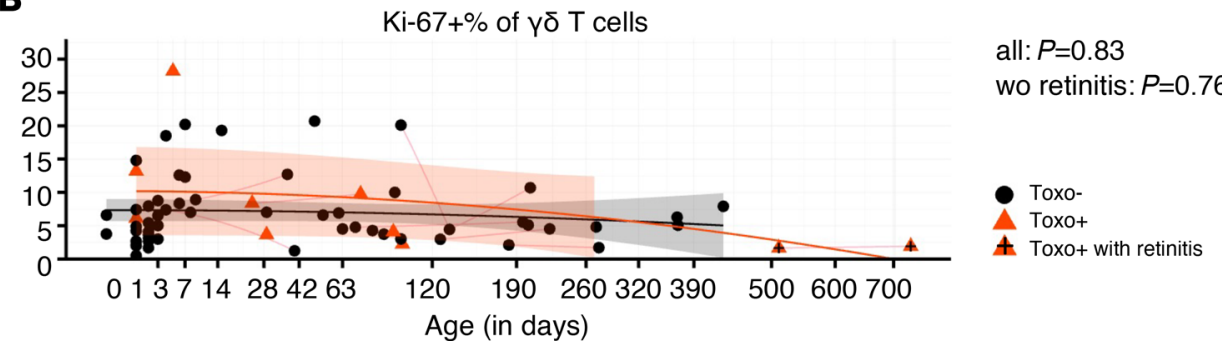

Figure 1. Congenital T. gondii infection induces expansion of $\gamma \delta$ T cells in utero. (A) Percentage of $\gamma \delta$ T cells (of total $\mathrm{CDB}^{+}$T cells) versus age (sample number: Toxo-: $n=66$, Toxo': $n=17$; subject number: $n=74$, Toxo $n=15$ ). (B) Expression of the proliferation marker Ki-67 in $\gamma \delta$ T cells versus age (sample number: Toxo $: n=55$, Toxo $: n=10$; subject number: $n=56$, $\operatorname{Toxo}^{+} n=8$ ). Toxo samples are indicated in orange triangles; Toxo- samples are indicated in black dots. Lines connect samples of the same subject. Samples from subjects with symptoms (retinitis) are indicated with plus signs. $P$ values of Toxo $^{+}$(with or without the subjects with symptoms) samples versus Toxosamples are calculated by Mann-Whitney $U$ test. The $95 \%$ confidence interval (linear model) of more than 0 area is indicated for each group (orange for $\mathrm{Toxo}^{+}$, gray for $\mathrm{Toxo}^{-}$).

Newborn Vy $9 V \delta 2$ T cells are highly differentiated upon congenital T. gondii infection. Next, we examined whether the expanded $\mathrm{V} \gamma 9 \mathrm{~V} \delta 2 \mathrm{~T}$ cells upon congenital toxoplasmosis were differentiated, as assessed by the downregulation of CD28 and CD27 (30-33). The V $\gamma 9 \mathrm{~V} \delta 2 \mathrm{~T}$ cells of $\mathrm{ToxO}^{+}$newborns were highly differentiated $\left(\mathrm{CD} 27^{-} \mathrm{CD} 28^{-}\right)$compared with Toxo $^{-}$newborns, while this was not the case for non- $\mathrm{V} \gamma 9 \mathrm{~V} \delta 2 \gamma \delta \mathrm{T}$ cells (Figure 3B). The few Toxo- newborns showing a CD28-CD27- phenotype (Figure $3 \mathrm{~A}$ ) could be due to an early response to the postnatal exposure to the microbiome $(34,35)$, but an influence of exposure to $T$. gondii-derived metabolites in utero cannot be excluded (36). At later ages, the difference between the $\mathrm{Toxo}^{+}$and $\mathrm{Toxo}^{-}$groups became less pronounced due to an increase in differentiated $\mathrm{V} \gamma 9 \mathrm{~V} \delta 2 \mathrm{~T}$ cells in Toxo- infants (Figure 3A). The level of HLA-DR expression, reflecting recent activation $(26,37)$, on $\mathrm{V} \gamma 9 \mathrm{~V} \delta 2 \mathrm{~T}$ cells, but not on non- $\mathrm{V} \gamma 9 \mathrm{~V} \delta 2 \mathrm{~T}$ cells, was also higher in the $\mathrm{Toxo}^{+}$group (Figure 3, C and D), although this was more subtle compared with the differences observed regarding the differentiation status (compare Figure 3A with Figure 3C). Thus, it appears that the $\mathrm{V} \gamma 9 \mathrm{~V} \delta 2 \mathrm{~T}$ cells were activated and differentiated in utero during the strong expansion, after which proliferation and activation declined while the differentiation status remained stable and high until early after birth.

Vy9V82 T cells express high levels of cytotoxic effector molecules upon congenital T. gondii infection. We investigated whether the differentiation of the $\mathrm{V} \gamma 9 \mathrm{~V} \delta 2 \mathrm{~T}$ cells in utero was associated with the expression of cytotoxic effector molecules, which can be important for fighting infections (38). While fetal $\mathrm{V} \gamma 9 \mathrm{~V} \delta 2 \mathrm{~T}$ cells express granzyme $\mathrm{A}(\mathrm{GzmA})$ in the absence of infection, they do not express granzyme B (GzmB) or perforin (10), the main cytotoxic effector molecules that can efficiently kill infected cells (38). Indeed, newborn $\mathrm{V} \gamma 9 \mathrm{~V} \delta 2 \mathrm{~T}$ cells of the Toxo $\mathrm{O}^{-}$group did not express $\mathrm{GzmB}$ or perforin (Figure 4, A-C). However, upon congenital T. gondii infection, the expression of these two cytotoxic mediators strikingly increased: a vast majority of (newborn) $\mathrm{V} \gamma 9 \mathrm{~V} \delta 2 \mathrm{~T}$ cells expressed $\mathrm{GzmB}$, while perforin was coexpressed in the $\mathrm{GzmB}^{\mathrm{hi}} \mathrm{V} \gamma 9 \mathrm{~V} \delta 2 \mathrm{~T}$ cells (Figure 4, A-C). The coexpression of GzmB and perforin is in line with the need of their combined actions to mediate their cytotoxic activity (38). At older ages, the $\mathrm{V} \gamma 9 \mathrm{~V} \delta 2 \mathrm{~T}$ cells of Toxo- infants started to also express GzmB and perforin (Figure 4, A and B). A relatively small difference in $\mathrm{GzmB}^{+}$and perforin ${ }^{+}$non- $\mathrm{V} \gamma 9 \mathrm{~V} \delta 2 \mathrm{~T}$ cells could be observed between 
Table 1. Clinical information regarding infants congenitally infected with $\mathrm{T}$. gondii

\begin{tabular}{|c|c|c|c|c|c|c|c|c|c|c|}
\hline Sample & Sample type & Sex & $\begin{array}{l}\text { Gestation } \\
\text { time at } \\
\text { diagnosis } \\
\text { (mother) }\end{array}$ & $\begin{array}{c}\text { Start of } \\
\text { treatment } \\
\text { (mother) }\end{array}$ & $\begin{array}{c}\text { Treatment } \\
\text { (mother) }\end{array}$ & $\begin{array}{l}\text { Age at } \\
\text { diagnosis } \\
\text { (infant) }\end{array}$ & $\begin{array}{l}\text { Start of } \\
\text { treatment } \\
\text { (infant) }\end{array}$ & $\begin{array}{l}\text { Treatment } \\
\text { (infant) }\end{array}$ & $\begin{array}{l}\text { Symptoms } \\
\text { (infant) }\end{array}$ & $\begin{array}{c}\text { Age at } \\
\text { sampling }\end{array}$ \\
\hline I-Toxo-011 & FACS-lysed & $\mathrm{F}$ & $28 \mathrm{gw}$ & $29 \mathrm{gw}$ & Pyr+sulf & At birth & 3 weeks & Pyr+sulf+FA & No & 1 day \\
\hline I-Toxo-012 & FACS-lysed & $\mathrm{F}$ & $34 \mathrm{gw}$ & $34 \mathrm{gw}$ & Spyr & At birth & 3 weeks & Pyr+sulf+FA & No & 1 day \\
\hline I-Toxo-075 & FACS-lysed & $\mathrm{F}$ & $26 \mathrm{gw}$ & $28 \mathrm{gw}$ & Pyr+sulf & In utero & At birth & Pyr+sulf+FA & No & 29 days \\
\hline I-Toxo-080 & $\begin{array}{c}\text { FACS-lysed/ } \\
\text { PBMC }\end{array}$ & $M$ & $24 \mathrm{gw}$ & $26 \mathrm{gw}$ & Spyr & 4 months & 4 months & Pyr+sulf+FA & Retinitis & 511 days \\
\hline I-Toxo-085 & $\begin{array}{l}\text { FACS-lysed/ } \\
\text { PBMC }\end{array}$ & $\mathrm{F}$ & $35 \mathrm{gw}$ & $36 \mathrm{gw}$ & Spyr & At birth & 1 month & Pyr+sulf+FA & No & 73 days \\
\hline I-Toxo-90 & PBMC & $\mathrm{F}$ & $12 \mathrm{gw}$ & No & No & At birth & 3 months & Pyr+sulf+FA & No & 434 days \\
\hline I-Toxo-132 & PBMC & $\mathrm{F}$ & $33 \mathrm{gw}$ & - & no & At birth & 15 days & Pyr+sulf & No & 65 days \\
\hline I-Toxo-135 & PBMC & M & $22 \mathrm{gw}$ & $26 \mathrm{gw}$ & Spyr & At birth & 15 days & Pyr+sulf & No & 59 days \\
\hline I-Toxo-137 & PBMC & M & $25 \mathrm{gw}$ & $27 \mathrm{gw}$ & $\begin{array}{c}\text { Spyr/ } \\
\text { pyr+sulf }\end{array}$ & At birth & 15 days & Pyr+sulf & Retinitis & 67 days \\
\hline
\end{tabular}

All deliveries were term deliveries ( $>37 \mathrm{gw}$ ). All newborns were born in Italy; 10 mothers were from European and 5 from African origin. gw, gestation week; -, no information; pyr, pyrimethamine; sulf, sulfodiazine; spyr, spyramicin; FA, folic acid.

$\mathrm{ToxO}^{+}$and $\mathrm{Toxo}^{-}$subjects, possibly due to a bystander effect caused by cytokine production in the local environment (Supplemental Figure 4, A and B). Since the T-box family transcription factors T-bet and eomesodermin (eomes) can be important for the expression of GzmB and/or perforin (38), we investigated the expression of these transcription factors in $\mathrm{V} \gamma 9 \mathrm{~V} \delta 2 \mathrm{~T}$ cells. While the expression of T-bet followed the same expression pattern as GzmB and perforin (Figure 4D), this was not observed for eomes (Figure 4E). Granulysin is a cytotoxic granule pore-forming peptide that can permeabilize bacteria and parasites directly and deliver death-inducing GzmB into these pathogens (39). Furthermore, adult V $\gamma 9 \mathrm{~V} \delta 2 \mathrm{~T}$ cells, which are expanded in the blood stage of malaria-infected patients (Plasmodium falciparum), are able to reduce parasite reinvasion in a granulysin-dependent manner $(40,41)$. However, in contrast to GzmB and perforin, granulysin remained low in Toxo ${ }^{+}$infants, even at older ages (Figure $4 \mathrm{~F}$ ), indicating that this cytotoxic mediator does not play an important role in the defense of fetal V $\gamma 9 \mathrm{~V} \delta 2 \mathrm{~T}$ cells against congenital T. gondii infection. Finally, we confirmed the programmed expression of GzmA (10) in Toxo newborns, which was further increased by congenital T. gondii infection (Figure 4G).

In order to have a global overview of all the flow cytometry data of $\mathrm{V} \gamma 9 \mathrm{~V} \delta 2 \mathrm{~T}$ cells in $\mathrm{Toxo}^{+}$and Toxo- infants and how they compare to the data obtained in non-V $\gamma 9 \mathrm{~V} \delta 2 \mathrm{~T}$ cells and conventional $\alpha \beta$ $\mathrm{T}$ cells, we performed $\mathrm{t}$-distributed stochastic neighbor embedding (t-SNE) and principal components analysis (PCA). This analysis revealed that early after birth $\mathrm{V} \gamma 9 \mathrm{~V} \delta 2 \mathrm{~T}$ cells from $\mathrm{ToxO}^{+}$infants were clearly forming a distinct cytotoxic effector-related cluster, while this was not the case for non-V $\gamma 9 \mathrm{~V} \delta 2$ $\mathrm{T}$ cells and $\alpha \beta \mathrm{T}$ cells (Figure $4 \mathrm{H}$ and Supplemental Figure 5). Later in life, the V $\gamma 9 \mathrm{~V} \delta 2 \mathrm{~T}$ cells from $\mathrm{Toxo}^{-}$and $\mathrm{Toxo}^{+}$infants grouped together into 1 cluster (Figure $4 \mathrm{H}$ and Supplemental Figure 5). Thus, this global analysis highlights the early and potent response of $\mathrm{V} \gamma 9 \mathrm{~V} \delta 2 \mathrm{~T}$ cells toward congenital $T$. gondii infection, including the acquisition of a high coexpression of the cytotoxic effector molecules GzmB and perforin.

The Vy $9 V \delta 2$ TCR repertoire of Toxo infants contains a fetal footprint. To address whether the observed expansion of $\mathrm{V} \gamma 9 \mathrm{~V} \delta 2 \mathrm{~T}$ cells upon congenital toxoplasmosis (Figures 1 and 2 and Supplemental Figures 1 and 2) 
A CD3+ cells
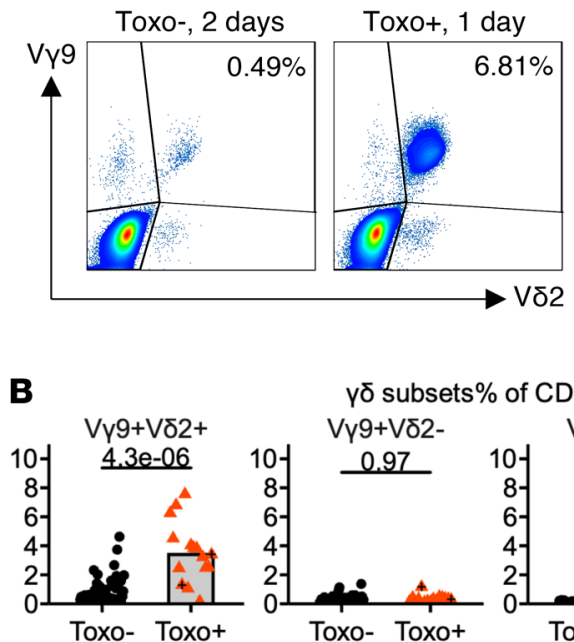

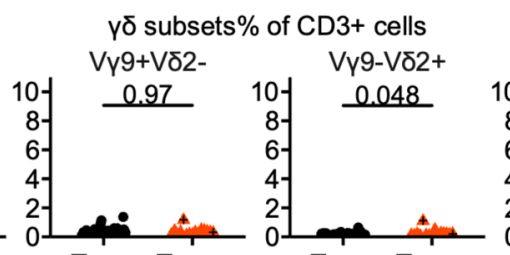

Toxo- Toxo+

Toxo- Toxo+

wo retinitis: $P=1.1 \mathrm{e}-05 / 0.66 / 0.15 / 0.24$

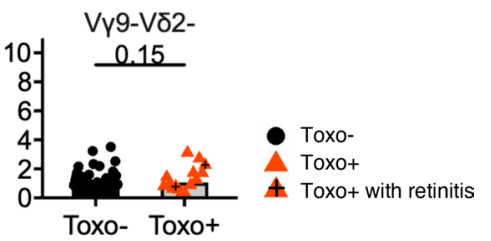

Toxo- $\mathrm{ToxO}^{+}$

C

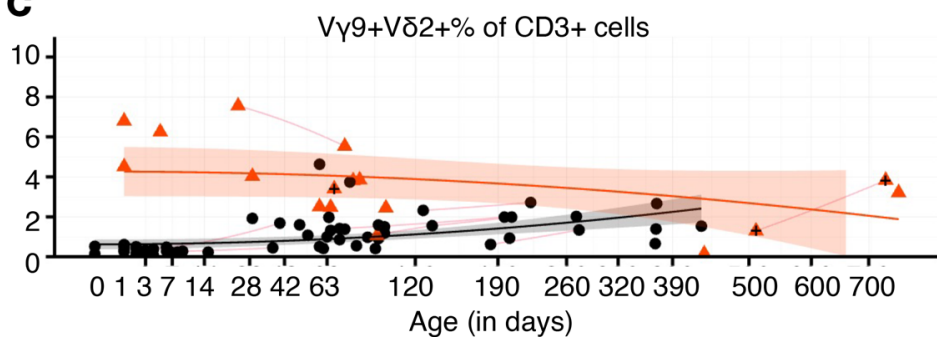

Toxo-

Toxo+

4 Toxo+ with retinitis

Figure 2. The expansion of $\gamma \delta \mathrm{T}$ cells upon congenital $T$. gondii infection is highly restricted to $\mathbf{V} \gamma \mathbf{9}^{+} \mathbf{V} \delta \mathbf{2}^{+} \mathrm{T}$ cells. (A) Representative flow cytometry plots of a Toxo- (2 days old) and a Toxo+ $\left(1\right.$ day old) newborn. Gate is on $\mathrm{CD3}^{+} \mathrm{T} \mathrm{lympho-}$

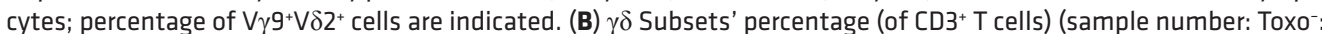
$n=66$, Toxo $: n=17$; subject number: $n=74$, Toxo $n=15$ ). (C) Percentage of $V_{\gamma} 9^{+} V \delta 2^{+}$cells (of total $C D 3^{+}$T cells) versus age (sample number: Toxo-: $n=66$, Toxo': $n=17$; subject number: $n=74$, Toxo $n=15$ ). Toxo samples are indicated in orange triangles; Toxo- samples are indicated in black dots. Lines connect samples of the same subject. Samples from subjects with symptoms (retinitis) are indicated with plus signs. $P$ values of Toxo (with or without the subjects with symptoms) samples versus Toxo- samples are calculated by Mann-Whitney $U$ test. The $95 \%$ confidence interval (linear model) of more than 0 area is indicated for each group (orange for $\mathrm{Toxo}^{+}$, gray for $\mathrm{Toxo}^{-}$).

shaped their TCR repertoire, we analyzed the CDR3 of the $\gamma$ and $\delta$ chain of sorted blood $\gamma \delta$ T cells of Toxo and Toxo ${ }^{-}$infants at 2 months after birth (the earliest age at which we had a sufficient amount of sample material at the right conditions for TCR repertoire analysis; Toxo $\left.{ }^{+} n=5, \operatorname{Toxo}^{-} n=10\right)$ and at 1 year $\left(\operatorname{Toxo}^{+}\right.$ $n=3$, Toxo $\left.^{-} n=4\right)$. At both age points, one of the Toxo ${ }^{+}$infants had symptoms (retinitis).

The random insertion of nucleotides (denoted by $\mathrm{N}$ ) by the enzyme TdT into the junctions of the joining V(D)J gene segments can significantly increase the junctional diversity of the CDR3 region (42). The level of TdT expression is low in fetal life, which is associated with the absence or a low number of $\mathrm{N}$ additions in the CDR3 repertoire of fetal $\gamma \delta \mathrm{T}$ cells $(17,43)$. Compared with Toxo infants, the TRGV9- and TRDV2-containing CDR3 repertoire of Toxo ${ }^{+}$infants at 2 months contained a lower number of $\mathrm{N}$ additions (Figure 5A), especially in the TRDV2-containing CDR3 sequences using TRDJ1 as a joining gene segment (Figure 5B). At 1 year, differences were less clear (Supplemental Figure 6, A and B). Note that the $\mathrm{Toxo}^{+}$infant showing symptoms had the highest number of $\mathrm{N}$ additions, especially in the TRDV2-containing CDR3 (Figure 5, A and B). The difference in $\mathrm{N}$ additions between Toxo ${ }^{+}$and Toxo ${ }^{-}$infants was specific for the TRDV2- and TRGV9-containing sequences (Supplemental Figure 6C), which is in line with the distinct expansion of $\mathrm{V} \gamma 9 \mathrm{~V} \delta 2 \mathrm{~T}$ cells upon congenital T. gondii infection (Figure 2 and Supplemental Figures 1 and 2)

The lower number of $\mathrm{N}$ additions in the TRGV9- and TRDV2-containing TCR repertoire of 2-month-old Toxo $^{+}$infants (with the notable exception of the infant with symptoms) indicates a fetal 
A

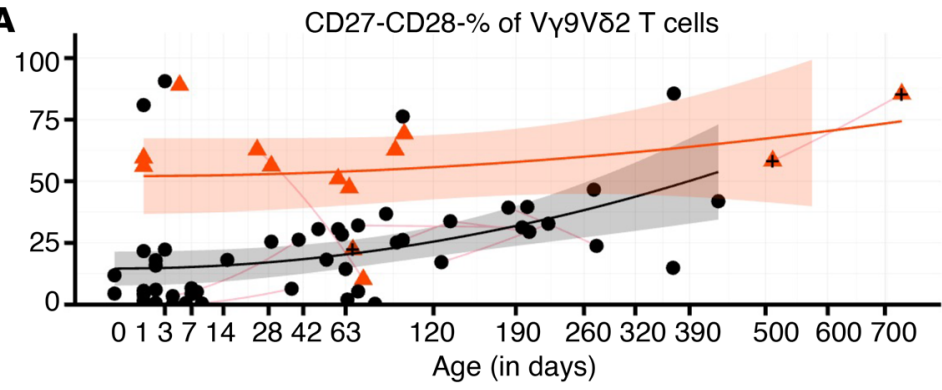

B

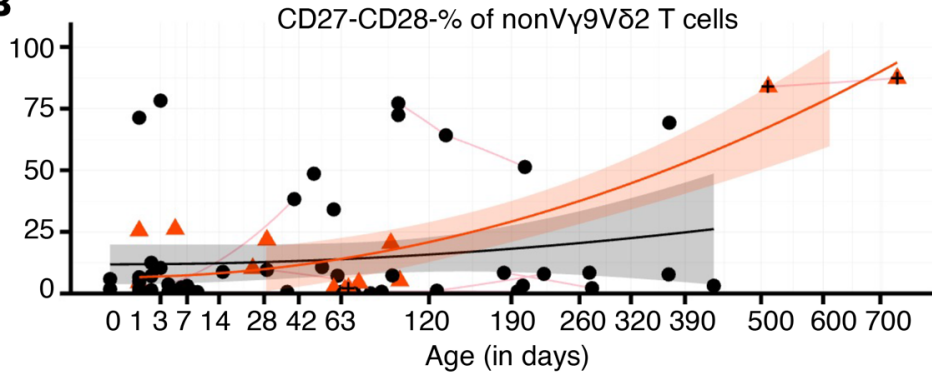

C

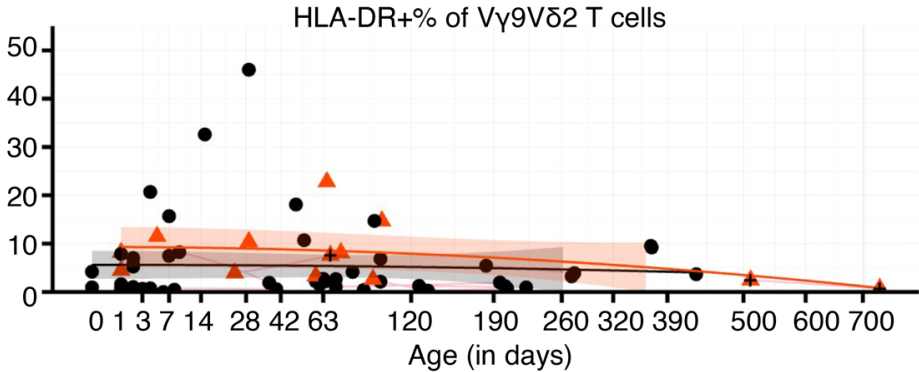

D

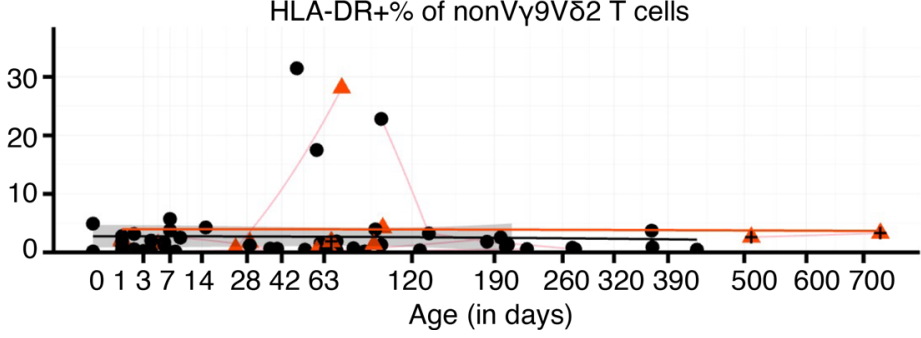

all: $P=5.7 \mathrm{e}-05$

wo retinitis: $P=7.9 \mathrm{e}-05$

Toxo-

Toxo+

4 Toxo+ with retinitis

all: $P=0.059$

wo retinitis: $P=0.094$

all: $P=0.027$

wo retinitis: $P=0.027$

all: $P=0.21$

wo retinitis: $P=0.34$

Toxo-
Toxo+
Aoxo+ with retinitis

Figure 3. $\mathbf{V} \gamma \mathbf{9 V} \delta 2 \mathrm{~T}$ cells are differentiated upon congenital T. gondii infection. (A) Percentage of CD27-CD28cells of $\mathrm{V} \gamma 9^{+} \mathrm{V} \delta 2^{+} \gamma \delta$ T cells versus age (sample number: Toxo- $n=53$, Toxo $: n=13$; subject number: $n=57$, Toxo $n=11$ ). (B) Percentage of $C D 27^{-} C D 28^{-}$cells of non $-\mathrm{V} \gamma 9^{+} \mathrm{V} \delta 2^{+} \gamma \delta$ T cells versus age (sample number: Toxo- $: n=53$, Toxo $: n=13$; subject number: $n=57$, Toxo $n=11$ ). (C) Percentage of HLA-DR+ cells of $\mathrm{V} \mathrm{g}^{+} \mathrm{V} \delta 2^{+} \gamma \delta$ T cells versus age (sample number: Toxo- $: n=53$, Toxo $: n=13$; subject number: $n=57$, Toxo $n=11$ ). (D) Percentage of HLA-DR cells of non- $V \gamma 9^{+} \mathrm{V} \delta 2^{+} \gamma \delta$ T cells versus age (sample number: Toxo- $n=53$, Toxo+ $: n=13$; subject number: $n=57$, $\left.\mathrm{Toxo}^{+} n=11\right)$. Toxo samples are indicated in orange triangles; Toxo- samples are indicated in black dots. Lines connect samples of the same subject. Samples from subjects with symptoms (retinitis) are indicated with plus signs. $P$ values of $\mathrm{Toxo}^{+}$(with or without the subjects with symptoms) samples versus Toxo- samples are calculated by Mann-Whitney $U$ test. The $95 \%$ confidence interval (linear model) of more than 0 area is indicated for each group (orange for $\mathrm{ToxO}^{+}$, gray for $\mathrm{Toxo}^{-}$).

origin since these features are known to be enriched in fetal blood V $\gamma 9 \mathrm{~V} \delta 2 \mathrm{~T}$ cells (17). To address this more directly, we investigated the overlap of the TRGV9 and TRDV2 CDR3 infant repertoires with the repertoires derived from fetal blood (22-30 weeks gestation), cord blood (39-41 weeks gestation), and adult blood (26-64 years) (17) (Supplemental Figure 7). Compared with Toxo- infants, the TRDV2 CDR3 repertoire of $\mathrm{Toxo}^{+}$infants, when excluding the "outlier" infant with symptoms (Figure 5A, "N addition") was shared more with the fetal blood repertoire (Supplemental Figure 7B, right panel). A tendency for such increased sharing was also observed when compared with term delivery cord blood 
A

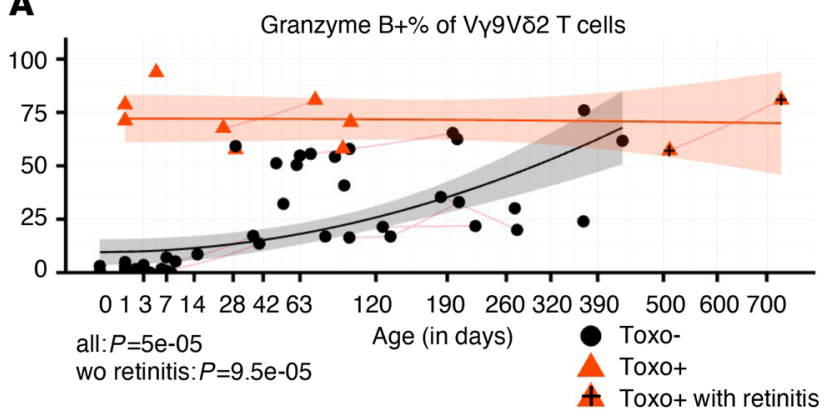

C

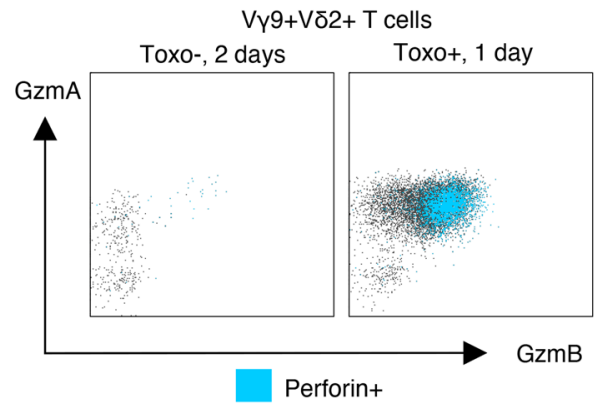

E

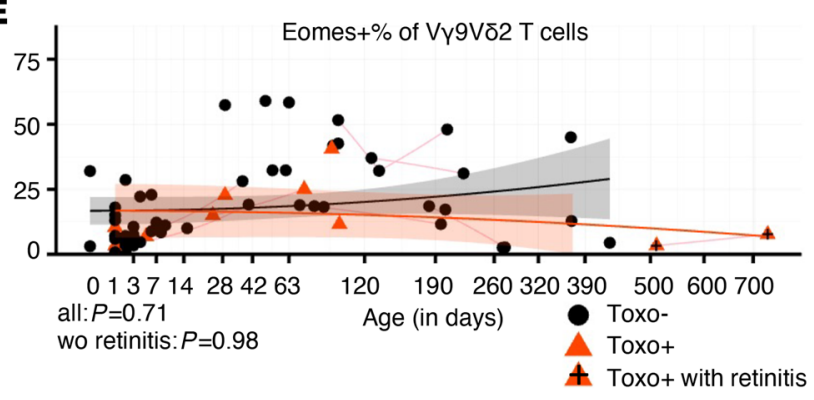

G

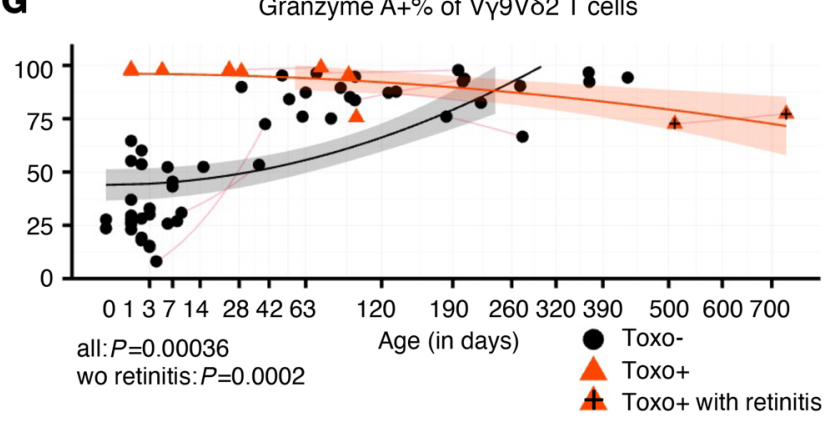

B

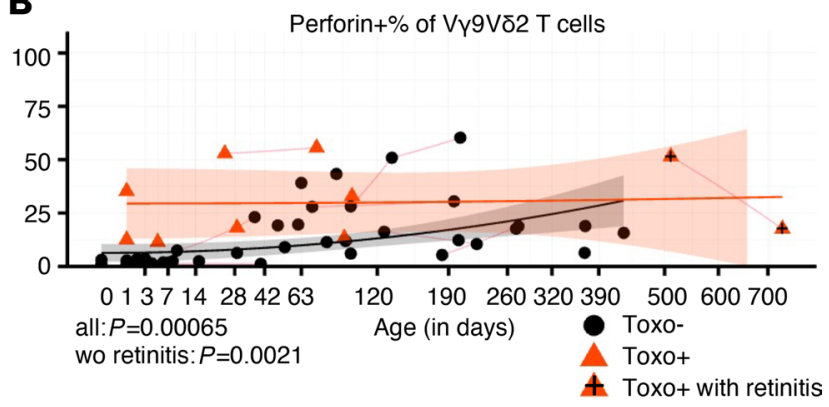

D $\quad$-bet+\% of VygVס2 $\mathrm{T}$ cells

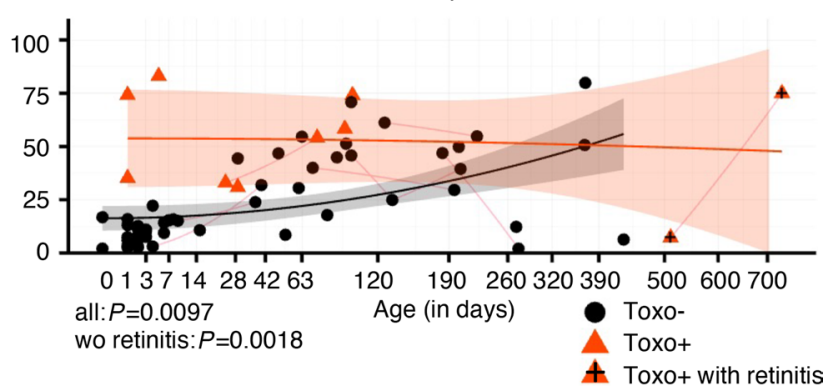

$\mathbf{F}$

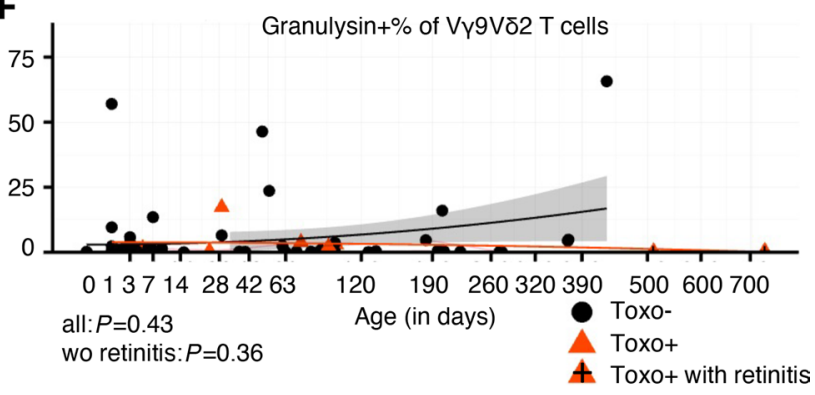

H

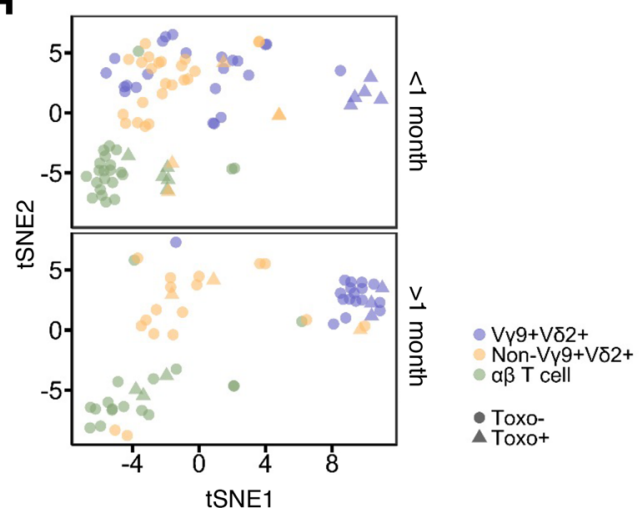

Figure 4. $\mathbf{\gamma} \gamma \mathbf{9 V} \delta 2 \mathrm{~T}$ cells express high levels of cytotoxic effector molecules upon congenital $T$. gondii infection. (A) Percentage of GzmB ${ }^{+}$cells of

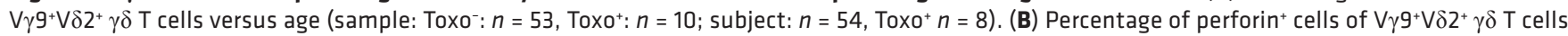
versus age (sample: Toxo-: $n=53$, Toxo+ $n=10$; subject: $n=54$, Toxo+ $n=8$ ). (C) Flow cytometry plots gated on $V \gamma 9 V \delta 2 \mathrm{~T}$ cells of a representative sample of Toxo- (2 days old) and Toxo+ (1 day old) newborns illustrating the expression of GzmA, GzmB, and perforin. (D) Percentage of T-bet ${ }^{+}$cells of $\mathrm{V} \mathrm{g}^{+} \mathrm{V} \delta 2^{+} \gamma \delta$ T cells versus age (sample: Toxo-: $n=55$, Toxo $: n=10$; subject: $n=56$, Toxo+ $n=8$ ). (E) Percentage of eomes ${ }^{+}$cells of $V \gamma 9^{+} \mathrm{V} \delta 2^{+} \gamma \delta \mathrm{T}$ cells versus age (sample: Toxo: $n=55$, Toxo $: n=10$; subject: $n=56$, Toxo ${ }^{+} n=8$ ). (F) Percentage of granulysin ${ }^{+}$cells of $\mathrm{V} \gamma 9^{+} \mathrm{V} \delta 2^{+} \gamma \delta \mathrm{T}$ cells versus age (sample: Toxo-: $n=53$, Toxo': $n=10$; subject: $n=54$, Toxo+ $n=8$ ). (G) Percentage of GzmA+ cells of $\mathrm{V} 9^{+} \mathrm{V} \delta 2^{+} \gamma \delta$ T cells versus age (sample: Toxo- $n=$ 53, Toxo $: n=10$; subject: $n=54$, Toxo $n=8$ ). (H) t-SNE analysis of flow cytometry results (11 markers [HLA-DR, CD27, CD28, CD45RA, Ki-67, T-bet, eomes, GzmA, GzmB, granulysin, perforin], $n=48$ subjects). Toxo+ samples are indicated in orange triangles; Toxo- samples are indicated in black dots. Lines connect samples of the same subject. Samples from subjects with symptoms (retinitis) are indicated with plus signs. $P$ values of Toxo ${ }^{+}$ (with or without the subjects with symptoms) samples versus Toxo- samples are calculated by Mann-Whitney $U$ test. The $95 \%$ confidence interval (linear model) of more than 0 area is indicated for each group (orange for Toxo ${ }^{+}$, gray for Toxo-). 
A
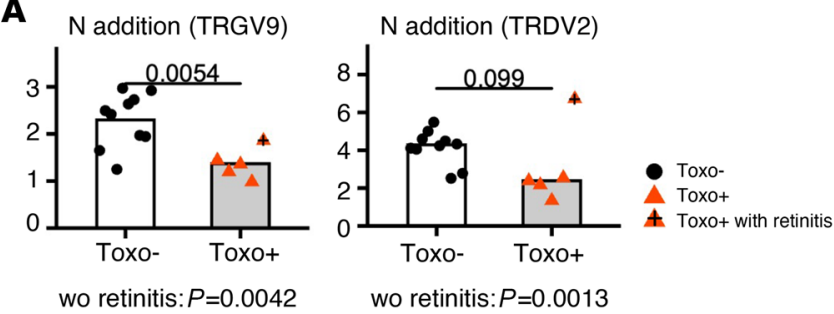

wo retinitis: $P=0.0013$

C

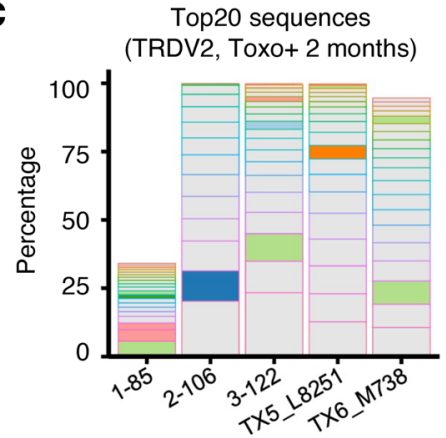

B

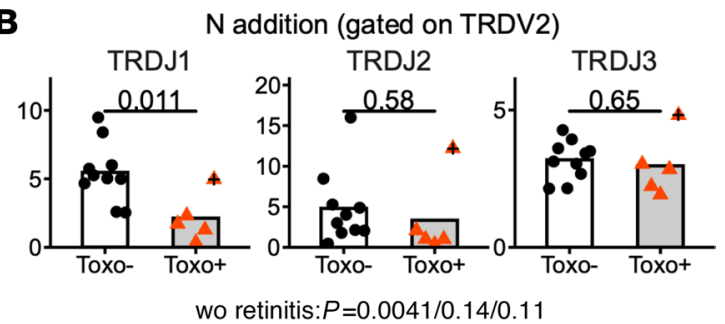

D

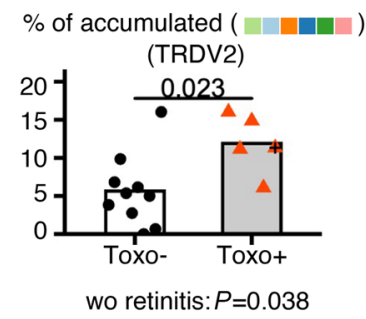

Figure 5. The $\mathbf{V} \gamma \mathbf{9 V} \delta \mathbf{2}$ TCR repertoire of Toxo+ newborns contains a fetal footprint. (A) Number of $\mathrm{N}$ additions of TRGV9- and TRDV2-containing CDR3 of sorted blood $\gamma \delta$ T cells from 2-month-old infants. $P$ values (indicated on the bar graphs) are obtained by the Student's $t$ test for TRGV 9 addition (bar indicates mean) and by Mann-Whitney $U$ test for TRDV2 $N$ addition (bar indicates median). (B) Number of $N$ additions of TRDV2-containing CDR3 using either TRDJ1, TRDJ2 or TRDJ3 of sorted blood $\gamma \delta$ T cells from 2-month-old infants. $P$ values (indicated on the bar graphs) are calculated by Student's $t$ test; bar indicates mean. (C) Accumulated percentage of the top 20 TRDV2-containing CDR3 sequences of 5 Toxo 2 -month-old samples (obtained from sorted blood $\gamma \delta$ T cells). Six germline-encoded sequences are indicated in different colors. (D) Accumulated percentage of the 6 germline-encoded sequences indicated in C in Toxo and Toxo- 2-month-old infants (sorted blood $\gamma \delta$ T cells). $P$ value (indicated on the bar graph) is calculated by Student's 2-tailed $t$ test; bar indicates mean. One subject with symptoms (retinitis) is indicated. The $P$ values obtained without this subject with symptom are indicated under each bar figure.

but was completely absent when compared with adult blood (Supplemental Figure 7B, right panel). The TRGV9 repertoire did not show such differences in sharing between $\mathrm{Toxo}^{+}$and $\mathrm{Toxo}^{-}$infants (Supplemental Figure 7B, left panel), which is probably due to the high prevalence of the public clonotype CALWEVQELGKKIKVF $(10,27,34)$ in both the Toxo ${ }^{-}$and Toxo $^{+}$group (Supplemental Figure 6D). These data indicate that the Toxo ${ }^{+}$TRDV2 repertoire at 2 months has an origin early in fetal life. Therefore, we verified the presence of early fetal germline-encoded (i.e., without $\mathrm{N}$ additions) TRDV2 CDR3 sequences $(21,34,43)$ in the top 20 clonotypes of the Toxo ${ }^{+}$infants (Figure $5 \mathrm{C}$ ) and found that their accumulated frequency was more prevalent in 2-month $\mathrm{Toxo}^{+}$compared with 2-month $\mathrm{Toxo}^{-}$ infants (Figure 5D). A possible explanation for the high prevalence of the CACDVLGDTDKLIF and CACDILGDTDKLIF TRDV2 CDR3 sequences in early fetal life $(21,34)$ is that the P nucleotide(s) needed to form these sequences can be derived from both the TRDV2 and the TRDD3 gene segment (Supplemental Figure 8). This can occur in an efficient way in the absence of $\mathrm{N}$ additions because of the low expression of the TdT enzyme in fetal life (43). In addition, there is a short-homology repeat present between the TRDD3 and TRDJ1 gene segments (Supplemental Figure 8). The prevalence of the third fetal liver sequence, CACDTGGYTDKLIF, can be explained by short-homology recombination (Supplemental Figure 8). Note that the short-homology repeat between TRDV2 and TRDD3 (the nucleotides $a c$ ) to form CACDTGGYTDKLIF has been described previously (43).

In summary, it appears that $\mathrm{V} \gamma 9 \mathrm{~V} \delta 2$ clonotypes with a fetal origin expand extensively in utero when faced with congenital $T$. gondii infection, resulting in a TCR repertoire footprint that is still present at 2 months after birth.

\section{Discussion}

Despite their high activation threshold in vitro, we show here that fetal $\mathrm{V} \gamma 9 \mathrm{~V} \delta 2 \mathrm{~T}$ cells can respond vigorously to a parasite infection in utero. A main finding of our study was the presence among congenital $\mathrm{Toxo}^{+}$infants of a fetal footprint in their $\gamma \delta \mathrm{TCR}$ repertoire (including germline-encoded TCR sequences), most likely because of the high expansion of fetal $\mathrm{V} \gamma 9 \mathrm{~V} \delta 2 \mathrm{~T}$ cells in utero. In line with these in vivo observations, $\mathrm{V} \gamma 9 \mathrm{~V} \delta 2 \mathrm{~T}$ cell clones expressing fetal germline-encoded TCR sequences are responsive in 
vitro toward phosphoantigen-containing mycobacterial extracts (21). Moreover, cord blood V $\gamma 9 \mathrm{~V} \delta 2 \mathrm{~T}$ cells proliferate upon incubation with T. gondii-infected PBMC in vitro (26). We propose that protection against phosphoantigen-generating pathogens, such as $T$. gondii, may have provided a selective pressure during evolution for the maintenance of the germline-encoded genetic elements needed for the generation of phosphoantigen-reactive TCRs early during fetal development $(17,19)$. This is in agreement with the high level of heritability of $\mathrm{V} \gamma 9 \mathrm{~V} \delta 2 \mathrm{~T}$ cells compared with other innate-like $\mathrm{T}$ cells (44). V $\gamma 9 \mathrm{~V} \delta 2 \mathrm{~T}$ cells from the older infants of the Toxo ${ }^{-}$group showed postnatal expansions, likely due to a more general phosphoantigen exposure (e.g., microbiome) $(34,35)$, thus reaching similar $\mathrm{V} \gamma 9 \mathrm{~V} \delta 2 \mathrm{~T}$ cell percentages as their $\mathrm{Toxo}^{+}$ counterparts. It is worth noting that the newborn who showed sequelae due to congenital $T$. gondii infection (retinitis) did not show a fetal footprint like the $\mathrm{Toxo}^{+}$infants without symptoms. Further studies are needed to investigate whether the lack of a fetal V $\gamma 9 \mathrm{~V} \delta 2 \mathrm{~T}$ cell footprint can be linked to the development of symptoms upon congenital $T$. gondii infection.

In contrast to our data, a previous study, mainly based on in vitro restimulation data, indicated that congenital T. gondii infection induces an anergic state in infant V $\gamma 9 \mathrm{~V} \delta 2 \mathrm{~T}$ cells (45). However, other $\mathrm{V} \gamma 9 \mathrm{~V} \delta 2$ $\mathrm{T}$ cell functions were not assessed and age-matched controls were lacking $(10,45-47)$. Our data indicate that a major wave of proliferation of fetal $\mathrm{V} \gamma 9 \mathrm{~V} \delta 2 \mathrm{~T}$ cells occurs in utero upon T. gondii encounter and is accompanied by the acquisition of the potent expression of cytotoxic mediators $\left(\mathrm{GzmB}^{+}\right.$perforin $\left.{ }^{+}\right)$. These effector functions can be used to kill T. gondii-infected cells, as illustrated by the in vitro study of Subauste et al. with $\mathrm{V} \gamma 9 \mathrm{~V} \delta 2 \mathrm{~T}$ cell lines and clones (26). Such a response that combines innate (germline-encoded TCR acting as a pathogen recognition receptor) and adaptive (high proliferation upon pathogen encounter) features has been referred to as "adaptate" biology (48). Hara et al. suggested that V $\gamma 9 \mathrm{~V} \delta 2 \mathrm{~T}$ cells are susceptible to anergy induction because of their extrathymic development (45). However, we have recently shown that the human thymus clearly contains $\mathrm{V} \gamma 9^{+} \mathrm{V} \delta 2^{+}$cells (17). Based on our data from the current study, we conclude that congenital $T$. gondii infection does not induce an anergic state of fetal $\mathrm{V} \gamma 9 \mathrm{~V} \delta 2 \mathrm{~T}$ cells but rather transforms them to lymphocytes with a potent cytotoxic phenotype that contributes to protection against infection by killing $T$. gondii-infected cells.

We have previously shown that fetal non- $\mathrm{V} \gamma \mathrm{V} \delta 2 \gamma \delta \mathrm{T}$ cells, such as the public $\mathrm{V} \gamma 8 \mathrm{~V} \delta 1 \mathrm{~T}$ cells, play a major role in the response toward congenital human cytomegalovirus (HCMV) infection (30). Together with the data of our current study, it appears that the human fetus is equipped with $\gamma \delta \mathrm{T}$ cell subsets that show a division of labor in their response to congenital infections: (i) the $\mathrm{V} \gamma 9 \mathrm{~V} \delta 2 \mathrm{~T}$ cells that respond to T. gondii and possibly other phosphoantigen-generating pathogens and (ii) the non-V $\gamma 9 \mathrm{~V} \delta 2 \mathrm{~T}$ cells that target HCMV-infected cells. Data from human in vitro studies $(26,30)$ and in vivo studies in mice $(25,49,50)$ indicate that $\gamma \delta \mathrm{T}$ cells play a protective role against infections with T. gondii and HCMV, but it cannot be excluded that the potent effector $\gamma \delta$ $\mathrm{T}$ cells contribute to the development of pathologies observed upon congenital infections $(51,52)$.

A main correlate of protection of the malaria vaccine PfSPZ (attenuated Plasmodium falciparum sporozoite) are $\mathrm{V} \gamma 9 \mathrm{~V} \delta 2 \mathrm{~T}$ cells $(53,54)$. Our data, showing the importance of $\mathrm{V} \gamma 9 \mathrm{~V} \delta 2 \mathrm{~T}$ cells in the response toward congenital $T$. gondii infection, indicate that vaccines, or other strategies, could be developed targeting these cells to protect infants against (congenital T. gondii) infections. Tools to manipulate V $\gamma 9 \mathrm{~V} \delta 2$ $\mathrm{T}$ cells in vivo are becoming increasingly available and include modified phosphoantigens with improved pharmacological characteristics and monoclonal antibodies targeting BTN3A1 $(55,56)$. Both Plasmodium falciparum and T. gondii contain an organelle, the apicoplast, which has specific metabolic functions including the MEP pathway of isoprenoid synthesis. In this pathway, the metabolite HMBPP, the most potent natural phosphoantigen, is generated $(4,57)$. This indicates that T. gondii-derived HMBPP is a major driving force for the expansion of fetal $\mathrm{V} \gamma 9 \mathrm{~V} \delta 2 \mathrm{~T}$ cells in utero. However, in contrast to our observations in congenital toxoplasmosis, Cairo et al. observed a depletion of phosphoantigen-reactive $\mathrm{V} \gamma 9 \mathrm{~V} \delta 2 \mathrm{~T}$ cells in placental malaria (27). A main difference between congenital T. gondii infection and placental malaria is that the malaria parasite very rarely crosses the placenta into the fetal circulation to establish an infection (58). Furthermore, the type of placental malaria infection can have opposing effects on the immune system in early life, thus possibly contributing to the differential effect on the fetal V $\gamma 9 \mathrm{~V} \delta 2 \mathrm{~T}$ cells $(27,58)$.

In immunocompromised (adult) patients (AIDS and transplant patients), toxoplasmosis is a major cause of morbidity and mortality (23). HIV infection leads to a decrease of V $\gamma 9 \mathrm{~V} \delta 2 \mathrm{~T}$ cells (59), but it is not clear to what extent the depletion of these potential $T$. gondii-responsive cells contributes to $T$. gondii-induced morbidities. HCMV infection is a major driving force of non-V $\gamma 9 \mathrm{~V} \delta 2 \gamma \delta \mathrm{T}$ cell expansion in organ transplant and hematopoietic stem cell transplant patients (60-62), and these expansions are associated with reduced 
cancer development $(63,64)$. In contrast, the role of $T$. gondii infection in driving V $\gamma 9 \mathrm{~V} \delta 2 \mathrm{~T}$ cell expansion/ differentiation in these transplant settings and their potential anticancer role is not known. Therefore, the role of $\mathrm{V} \gamma 9 \mathrm{~V} \delta 2 \mathrm{~T}$ cells in toxoplasmosis in transplant and AIDS patients deserves further investigation.

Overall, our data indicate that the human fetus, from early gestation onward, possesses V $\gamma 9 \mathrm{~V} \delta 2 \mathrm{~T}$ cells that can expand and transform into killer effector cells upon congenital T. gondii infection. Thus, these fetal innate $\mathrm{T}$ cells could provide protection against parasite infections in utero.

\section{Methods}

Blood sample collection and processing. Peripheral blood was collected in the Microbiology and Virology outpatient center of IRCCS San Matteo Hospital Foundation, Pavia, Italy. The diagnosis of congenital toxoplasmosis was performed with Liaison XL Toxo IgG II / IgM CLIA, Novalisa IgA (DiaSorin), VIDAS Toxo IgG II, ISAGA IgM (bioMérieux), IgG-IgM Western blot (LDBio), and homemade interferon- $\gamma$ release assay (65). IGRA test was performed in the same way as the test developed by Chapey and colleagues (65) with some modification, i.e., the antigen employed (the same antigen utilized for Liaison commercial tests) with a final concentration of $3 \mu \mathrm{g} / \mathrm{mL}$, which was provided by DiaSorin. This concentration yielded the best results according to previous studies. The ELISA test to evaluate interferon- $\gamma$ production is a commercially available kit (QIAGEN).

All the mothers (recruited from Italy, $n=72$ ) from both infected and noninfected groups were diagnosed with $T$. gondii infection during pregnancy and were treated in the same way (with spyramicin and/or pyrimethamine + sulfodiazine + folinic acid) (66). Depending on the volume of collected blood, samples were either directly lysed $(\sim 0.5 \mathrm{~mL})$ with FACS-lysing solution (BD FACS Lysing Solution) or processed $(\sim 1 \mathrm{~mL})$ to isolate PBMCs with Lymphoprep gradient centrifugation (Lymphoprep, STEMCELL Technologies) and stored in liquid nitrogen. Frozen PBMC samples and FACS-lysed blood samples were then sent to the Institute for Medical Immunology of the ULB in Belgium.

Flow cytometry and antibody reagents. FACS-lysed samples were thawed from liquid nitrogen at $37^{\circ} \mathrm{C}$ and washed with PBS containing $0.1 \%$ bovine serum albumin (BSA) (MilliporeSigma). For surface staining, cells were incubated with antibody mix at $4^{\circ} \mathrm{C}$ for $15-20$ minutes, then washed and resuspended with $0.1 \%$ BSA/PBS. For intracellular staining, after surface staining, the Perm 2 kit (BD Biosciences) was used to permeabilize cell membranes. All samples were acquired either on CyAn ADP cytometer (Dako Cytomation) or LSRFortessa (BD Biosciences); analysis was done using FlowJo software and R.

The following antibodies were used in this study: CD3-PB (clone SP34-2, BD Biosciences), CD3-BV510 (UCHT1, BD Biosciences), TCR $\gamma \delta$-APC (11F2, Miltenyi Biotec), TCR V $\gamma 9$-PC5 (IMMU 360, Beckman Coulter), TCR V82-FITC (IMMU 389, Beckman Coulter), CD27-PE (M-T271, BD Biosciences), CD28ECD (CD28.2, Beckman Coulter), CD45RA-PC7 (L48, BD Biosciences), HLA-DR-V450 (G46-6, BD Biosciences), Ki-67-PC7 (B56, BD Biosciences), T-bet-BV421 (4B10, BioLegend), eomes-PE (WD1928, eBioscience, Thermo Fisher Scientific), granzyme A-PB (CB9, BioLegend), granzyme B-PE-CF594 (GB11, BD Biosciences), granulysin-PE (eBioDH2 [DH2], Invitrogen, Thermo Fisher Scientific), and perforin-PC7 (dG9, delta G9; eBioscience, Thermo Fisher Scientific).

Cell sorting, RNA isolation, and CDR3 analysis. For PBMC samples, cells were thawed at $37^{\circ} \mathrm{C}$ in complete medium [RPMI 1640 from Gibco, Thermo Fisher Scientific, supplemented with L-glutamine ( $2 \mathrm{mM})$, penicillin $(50 \mathrm{U} / \mathrm{mL})$, streptomycin $(50 \mathrm{U} / \mathrm{mL})$, and $1 \%$ nonessential amino acids from Lonza and $10 \%$ (v/v) heat-inactivated FCS from PPA Laboratories], then labeled with Zombie NIR dye (BioLegend) at room temperature for 10 minutes and stained with CD3/TCR $\gamma \delta / \mathrm{TCR}$ V $\gamma 9 / \mathrm{TCR}$ V $\delta 2$ antibodies at $4^{\circ} \mathrm{C}$ for 15 minutes. $\mathrm{CD}^{+} \mathrm{TCR} \gamma \delta^{+} \mathrm{T}$ cells were sorted on FACSAria III (BD Biosciences) with a mean purity of $98.0 \%$. Cells were snap-frozen in liquid nitrogen and preserved in $-80^{\circ} \mathrm{C}$.

RNA was isolated from sorted $\gamma \delta$ T cells ( 10,000 cells) with the RNeasy Micro Kit (QIAGEN). cDNA was generated by performing a template-switch anchored reverse transcription PCR. RNA was reverse-transcribed via a template-switch cDNA reaction using TRGC-specific (5'-CAAGAAGACAAAGGTATGTTCCAG-3') and TRDC-specific (5'-GTAGAATTCCTTCACCAGACAAG-3') primers in the same reaction tube, a template-switch adapter (5'-AAGCAGTGGTATCAACGCAGAGTACATrGrGrG-3'), and the Superscript II RT enzyme (Invitrogen, Thermo Fisher Scientific). The TRGC primer binds both TRGC1 and TRGC2. The cDNA was then purified using AMPure XP Beads (Agencourt). Amplification of the TRG and TRD region was achieved using a specific TRGC primer (binding both TRGC1 and TRGC2 5'-GTCTCGTGGGCTCGGAGATGTGTATAAGAGACAGAATAGTGGGCTTGGGGGAAACATCTGCAT-3', adapter underlined) and a specific TRDC 
primer (5'-GTCTCGTGGGCTCGGAGATGTGTATAAGAGACAGACGGATGGTTTGGTATGAGGCTGACTTCT-3', adapter underlined) and a primer complementary to the template-switch adapter (5'-TCGTCGGCAGCGTCAGATGTGTATAAGAGACAGAAGCAGTGGTATCAACGCA G-3', adapter underlined) with the KAPA Real-Time Library Amplification Kit (Kapa Biosystems). Adapters were required for subsequent sequencing reactions. After purification with AMPure XP beads, an index PCR with Illumina sequencing adapters was performed using the Nextera XT Index Kit. This second PCR product was again purified with AMPure XP beads. High-throughput sequencing of the generated amplicon products containing the TRG and TRD sequences was performed on an Illumina MiSeq platform using the V2 300 kit, with 150 base pairs (bp) at the $3^{\prime}$ end (read 2) and 150 bp at the $5^{\prime}$ end (read 1) (at the GIGA center, University of Liège, Belgium).

After passing the quality check using fastqc (version 0.11.8, http://www.bioinformatics.babraham. ac.uk/projects/fastqc/), raw sequencing reads from fastq files (read 1 and read 2) were aligned to reference $\mathrm{V}, \mathrm{D}$, and J genes from GenBank database specifically for "TRG" or "TRD" to build CDR3 sequences using the MiXCR software (version 3.0.3) (67). Default parameters were used except to assemble the TRDD gene segments where 3 instead of 5 consecutive nucleotides were applied as the assembly parameter. CDR3 sequences were then exported and analyzed using VDJtools software (version 1.2.1) using default settings in order to calculate the number of $\mathrm{N}$ additions, the CDR3 length, the length of $\mathrm{J}$ gene segments, and the level of clonotype sharing between different samples (68). Sequences out of frame and containing stop codons were excluded from the analysis. Files generated from VDJtools were uploaded into Rstudio (version 1.1.463, R version 3.5.2), and analysis involved the following packages: ggplot2, dplyr, reshape, ggpubr, and ggseqlogo. Fastq files of TRG and TRD sequences are deposited in the Sequence Read Archive under accession number PRJNA625515.

Dimensionality reduction and clustering. Flow cytometry results generated from FlowJo and CDR3 data generated from VDJtools were uploaded into Rstudio. Packages ggfortify (https://CRAN.R-project.org/ package=ggfortify) and ggbiplot (http://github.com/vqv/ggbiplot) were used to generate PCA. Package Rtsne (https://github.com/jkrijthe/Rtsne) was used to generate t-SNE clustering analysis (69). Parameters were adapted according to sample size. t-SNE analyses were run multiple times using different parameters.

Statistics. Statistical analysis was done by using R. Student's 2-tailed $t$ test was used for normally distributed data (Shapiro-Wilk test, $P>0.05$ ) and with equal variances (Levene's test, $P>0.05$ ). Otherwise, Mann-Whitney $U$ test was used. When more than 1 blood sample was obtained from the same subject at different ages (e.g., Figure 1: in 8 out of 74 infants), only the earliest sample was used from this subject in the statistical test for the calculation of the $P$ value between Toxo $^{+}$and Toxo $^{-}$infants. For analysis of the data according to age, the linear model was used for prediction; the point-wise $95 \%$ confidence interval was indicated around the mean.

Study approval. This study was approved by IRCCS San Matteo Polyclinic Foundation ethical committee number 20160017812. All parents were provided with written and oral information about the study and gave their consent. Research was conducted in accordance with the Declaration of Helsinki.

\section{Author contributions}

LM, MP, and MT conducted experiments; FG and VM acquired crucial blood samples; AM, VM, and DV designed the study; LM and DV analyzed data; and DV wrote the manuscript.

\section{Acknowledgments}

We are grateful to all the mothers and infants for participating in this study. We thank the GIGA Genomics platform (Latifa Karim and Wouter Coppieters) for their outstanding technical support. This work was supported by the Fonds de la Recherche Scientifique (FNRS; CDR 0244.17) and the Fund John W. Mouton Pro Retina. LM is supported by the Chinese Scholarship Council, Fonds Van Buuren-Jaumotte-Demoulin, and Fonds Hoguet. MP is supported by the FNRS (FRIA and short-term postdoctoral fellowship), Fonds Van Buuren-Jaumotte-Demoulin, and Fonds Hoguet.

Address correspondence to: David Vermijlen, Department of Pharmacotherapy and Pharmaceutics, Faculty of Pharmacy, Université Libre de Bruxelles (ULB), Campus Plaine, Boulevard du Triomphe, Accès 2, 1050 Brussels, Belgium. Email: David.Vermijlen@ulb.be. 
1. Hayday AC. [gamma][delta] cells: a right time and a right place for a conserved third way of protection. Annu Rev Immunol. 2000;18:975-1026.

2. Silva-Santos B, et al. $\gamma \delta$ T cells in cancer. Nat Rev Immunol. 2015;15(11):683-691.

3. Vermijlen D, et al. $\gamma \delta$ T cell responses: how many ligands will it take till we know? Semin Cell Dev Biol. 2018;84:75-86.

4. Eberl M, et al. Microbial isoprenoid biosynthesis and human gammadelta T cell activation. FEBS Lett. 2003;544(1-3):4-10.

5. Morita CT, et al. Nonpeptide antigens, presentation mechanisms, and immunological memory of human Vgamma2Vdelta2 T cells: discriminating friend from foe through the recognition of prenyl pyrophosphate antigens. Immunol Rev. 2007;215:59-76.

6. Wang H, et al. Vgamma2Vdelta2 $\mathrm{T}$ cell receptor recognition of prenyl pyrophosphates is dependent on all CDRs. J Immunol. 2010;184(11):6209-6222.

7. Wang L, et al. Antibacterial effect of human V gamma 2V delta 2 T cells in vivo. J Clin Invest. 2001;108(9):1349-1357.

8. Tu W, et al. The aminobisphosphonate pamidronate controls influenza pathogenesis by expanding a gammadelta $\mathrm{T}$ cell population in humanized mice. J Exp Med. 2011;208(7):1511-1522.

9. Kobayashi H, Tanaka Y. $\gamma \delta$ T cell immunotherapy-a review. Pharmaceuticals (Basel). 2015;8(1):40-61.

10. Dimova T, et al. Effector V $\gamma 9 \mathrm{~V} \delta 2 \mathrm{~T}$ cells dominate the human fetal $\gamma \delta \mathrm{T}$-cell repertoire. Proc Natl Acad Sci U S A. 2015;112(6):E556-E565

11. Cairo C, et al. V delta 2 T-lymphocyte responses in cord blood samples from Italy and Cote d'Ivoire. Immunology. 2008;124(3):380-387.

12. Hsu H, et al. Prolonged PD1 expression on neonatal V $\delta 2$ lymphocytes dampens proinflammatory responses: role of epigenetic regulation. J Immunol. 2016;197(5):1884-1892.

13. Hong DK, Lewis DB. Developmental Immunology and Role of Host Defenses in Fetal and Neonatal Susceptibility to Infection. In: Wilson CB, Nizet V, Maldonado YA, Remington JS, Klein JO, eds. Infectious Diseases of the Fetus and Newborn Infant. Elsevier; 2016:90-197.

14. Zhang X, et al. Unique aspects of the perinatal immune system. Nat Rev Immunol. 2017;17(8):495-507.

15. Dauby N, Marchant A. Fetal Infections: Immune Response to Infections during Fetal Life. In: Kilby MD, Johnson A, Oepkes D, eds. Fetal Therapy. Cambridge University Press; 2019:215-223.

16. Rackaityte E, Halkias J. Mechanisms of fetal T cell tolerance and immune regulation. Front Immunol. 2020;11:588.

17. Papadopoulou M, et al. TCR sequencing reveals the distinct development of fetal and adult human V $\gamma 9 \mathrm{~V} \delta 2 \mathrm{~T}$ cells. J Immunol. 2019;203(6):1468-1479.

18. Ribot JC, et al. $\gamma \delta$ T cells in tissue physiology and surveillance. Nat Rev Immunol. 2021;21(4):221-232.

19. Papadopoulou M, et al. Innate and adaptive $\gamma \delta$ T cells: how, when, and why. Immunol Rev. 2020;298(1):99-116.

20. Park J-E, et al. A cell atlas of human thymic development defines T cell repertoire formation. Science. 2020;367(6480):eaay3224.

21. McVay LD, Carding SR. Extrathymic origin of human gamma delta T cells during fetal development. J Immunol. 1996;157(7):2873-2882.

22. McVay LD, et al. The generation of human gammadelta $\mathrm{T}$ cell repertoires during fetal development. J Immunol. 1998;160(12):5851-5860.

23. Montoya JG, Liesenfeld O. Toxoplasmosis. Lancet. 2004;363(9425):1965-1976.

24. Peyron F, et al. Toxoplasmosis. In: Wilson CB, Nizet V, Maldonado YA, Remington JS, Klein JO eds. Infectious Diseases of the Fetus and Newborn Infant. Elsevier; 2016:951-1044.

25. Kasper LH, et al. Induction of gammadelta T cells during acute murine infection with Toxoplasma gondii. J Immunol. 1996;157(12):5521-5527.

26. Subauste CS, et al. Preferential activation and expansion of human peripheral blood gamma delta $\mathrm{T}$ cells in response to Toxoplasma gondii in vitro and their cytokine production and cytotoxic activity against T. gondii-infected cells. JClin Invest. 1995;96(1):610-619.

27. Cairo C, et al. Cord blood V $\gamma 2 \mathrm{~V} \delta 2 \mathrm{~T}$ cells provide a molecular marker for the influence of pregnancy-associated malaria on neonatal immunity. J Infect Dis. 2014;209(10):1653-1662.

28. Denkers EY, Gazzinelli RT. Regulation and function of T-cell-mediated immunity during Toxoplasma gondii infection. Clin Microbiol Rev. 1998;11(4):569-588.

29. Jordan KA, et al. Kinetics and phenotype of vaccine-induced CD8+ T-cell responses to Toxoplasma gondii. Infect Immun. 2009;77(9):3894-3901.

30. Vermijlen D, et al. Human cytomegalovirus elicits fetal gammadelta T cell responses in utero. J Exp Med. 2010;207(4):807-821.

31. Pitard V, et al. Long-term expansion of effector/memory Vdelta2-gammadelta T cells is a specific blood signature of CMV infection. Blood. 2008;112(4):1317-1324.

32. Ryan PL, et al. Heterogeneous yet stable V $\delta 2(+)$ T-cell profiles define distinct cytotoxic effector potentials in healthy human individuals. Proc Natl Acad Sci U S A. 2016;113(50):14378-14383.

33. Davey MS, et al. Clonal selection in the human V $\delta 1 \mathrm{~T}$ cell repertoire indicates $\gamma \delta \mathrm{TCR}$-dependent adaptive immune surveillance. Nat Commun. 2017;8:14760.

34. Papadopoulou M, et al. Fetal public V $\gamma 9 \mathrm{~V} \delta 2 \mathrm{~T}$ cells expand and gain potent cytotoxic functions early after birth. Proc Natl Acad Sci U S A. 2020;117(31):18638-18648.

35. Ravens S, et al. Microbial exposure drives polyclonal expansion of innate $\gamma \delta \mathrm{T}$ cells immediately after birth. Proc Natl Acad Sci US A. 2020;117(31):18649-18660.

36. Dauby N, et al. Uninfected but not unaffected: chronic maternal infections during pregnancy, fetal immunity, and susceptibility to postnatal infections. Lancet Infect Dis. 2012;12(4):330-340.

37. Dieli F, et al. Targeting human \{gamma\} delta\} T cells with zoledronate and interleukin-2 for immunotherapy of hormone-refractory prostate cancer. Cancer Res. 2007;67(15):7450-7457.

38. Chowdhury D, Lieberman J. Death by a thousand cuts: granzyme pathways of programmed cell death. Annu Rev Immunol. 2008;26:389-420.

39. Dotiwala F, Lieberman J. Granulysin: killer lymphocyte safeguard against microbes. Curr Opin Immunol. 2019;60:19-29.

40. Farouk SE, et al. Gamma delta T cells inhibit in vitro growth of the asexual blood stages of Plasmodium falciparum by a granule 
exocytosis-dependent cytotoxic pathway that requires granulysin. Eur J Immunol. 2004;34(8):2248-2256.

41. Costa G, et al. Control of Plasmodium falciparum erythrocytic cycle: $\gamma \delta$ T cells target the red blood cell-invasive merozoites. Blood. 2011;118(26):6952-6962.

42. Chien YH, Konigshofer Y. Antigen recognition by gammadelta T cells. Immunol Rev. 2007;215:46-58

43. Tieppo P, et al. The human fetal thymus generates invariant effector $\gamma \delta$ T cells. J Exp Med. 2020;217(3):jem.20190580.

44. Mangino M, et al. Innate and adaptive immune traits are differentially affected by genetic and environmental factors. Nat Commun. 2017;8:13850.

45. Hara T, et al. Human V delta 2+ gamma delta T-cell tolerance to foreign antigens of Toxoplasma gondii. Proc Natl Acad Sci U S A. 1996;93(10):5136-5140.

46. Lewis DB, Wilson CB. Developmental immunology and role of host defenses in fetal and neonatal susceptibility to infection. In: Remington JS, Klein JO, eds. Infectious Disease of the Fetus and Newborn Infant. Elsevier; 2006:87-210.

47. Parker CM, et al. Evidence for extrathymic changes in the T cell receptor gamma/delta repertoire. J Exp Med. 1990;171(5):1597-1612.

48. Hayday AC. $\gamma \delta$ T cell update: adaptate orchestrators of immune surveillance. J Immunol. 2019;203(2):311-320.

49. Khairallah C, et al. $\gamma \delta$ T cells confer protection against murine cytomegalovirus (MCMV). PLoS Pathog. 2015;11(3):e1004702.

50. Sell S, et al. Control of murine cytomegalovirus infection by $\gamma \delta$ T cells. PLoS Pathog. 2015;11(2):e1004481.

51. Machado AS, et al. Biomarker analysis revealed distinct profiles of innate and adaptive immunity in infants with ocular lesions of congenital toxoplasmosis. Mediators Inflamm. 2014;2014:910621.

52. Huygens A, et al. Immunity to cytomegalovirus in early life. Front Immunol. 2014;5:552.

53. Ishizuka AS, et al. Protection against malaria at 1 year and immune correlates following PfSPZ vaccination. Nat Med. 2016;22(6):614-623.

54. Zaidi I, et al. $\gamma \delta \mathrm{T}$ cells are required for the induction of sterile immunity during irradiated sporozoite vaccinations. J Immunol. 2017;199(11):3781-3788.

55. Lentini NA, et al. Phosphonamidate prodrugs of a butyrophilin ligand display plasma stability and potent $\mathrm{V} \gamma 9 \mathrm{~V} \delta 2 \mathrm{~T}$ cell stimulation. J Med Chem. 2018;61(19):8658-8669.

56. Harly C, et al. Key implication of CD277/butyrophilin-3 (BTN3A) in cellular stress sensing by a major human $\gamma \delta$ T-cell subset. Blood. 2012;120(11):2269-2279.

57. Sheiner L, et al. The metabolic roles of the endosymbiotic organelles of Toxoplasma and Plasmodium spp. Curr Opin Microbiol. 2013;16(4):452-458.

58. Feeney ME. The immune response to malaria in utero. Immunol Rev. 2020;293(1):216-229.

59. Pauza CD, et al. $\gamma \delta$ T cells in HIV disease: past, present, and future. Front Immunol. 2015;5:687.

60. Déchanet J, et al. Implication of gammadelta $\mathrm{T}$ cells in the human immune response to cytomegalovirus. J Clin Invest. 1999;103(10):1437-1449.

61. Knight A, et al. The role of V $\delta 2$-negative $\gamma \delta \mathrm{T}$ cells during cytomegalovirus reactivation in recipients of allogeneic stem cell transplantation. Blood. 2010;116(12):2164-2172.

62. Scheper W, et al. $\gamma \delta \mathrm{T}$ cells elicited by CMV reactivation after allo-SCT cross-recognize CMV and leukemia. Leukemia. 2013;27(6):1328-1338.

63. Couzi L, et al. Cytomegalovirus-induced gammadelta T cells associate with reduced cancer risk after kidney transplantation. J Am Soc Nephrol. 2010;21(1):181-188.

64. Arruda LCM, et al. Impact of $\gamma \delta \mathrm{T}$ cells on clinical outcome of hematopoietic stem cell transplantation: systematic review and meta-analysis. Blood Adv. 2019;3(21):3436-3448.

65. Ciardelli L, et al. Early and accurate diagnosis of congenital toxoplasmosis. Pediatr Infect Dis J. 2008;27(2):125-129.

66. Peyron F, et al. Maternal and congenital Toxoplasmosis: diagnosis and treatment recommendations of a French multidisciplinary working group. Pathogens. 2019;8(1):24

67. Bolotin DA, et al. MiXCR: software for comprehensive adaptive immunity profiling. Nat Methods. 2015;12(5):380-381.

68. Shugay M, et al. VDJtools: unifying post-analysis of T cell receptor repertoires. PLoS Comput Biol. 2015;11(11):e1004503.

69. Van der Maaten L, Hinton G. Visualizing data using t-SNE. J Mach Learn Res. 2008;9:2579-2605. 